\title{
Gamification as a Sustainable Source of Enjoyment During Balance and Gait Exercises
}

\begin{abstract}
Katinka van der Kooij ${ }^{1 *}$, Rosanne van Dijsseldonk ${ }^{1,2}$, Milou van Veen ${ }^{1}$, Frans Steenbrink ${ }^{3}$, Coen de Weerd ${ }^{3}$ and Krista E. Overvliet ${ }^{4,5}$
\end{abstract}

'Department of Human Movement Sciences, Vrije Universiteit Amsterdam, Amsterdam, Netherlands, ${ }^{2}$ Department of Research, Sint Maartenskliniek, Nijmegen, Netherlands, ${ }^{3}$ Motekforce Link, Amsterdam, Netherlands, ${ }^{4}$ Department of Biological Psychology and Neuropsychology, University of Hamburg, Hamburg, Germany, ${ }^{5}$ Department of Experimental Psychology, Utrecht University, Utrecht, Netherlands

We may be motivated to engage in a certain motor activity because it is instrumental to obtaining reward (e.g., money) or because we enjoy the activity, making it intrinsically rewarding. Enjoyment is related to intrinsic motivation which is considered to be a durable form of motivation. Therefore, many rehabilitation programs aim to increase task enjoyment by adding game elements ("gamification"). Here we ask how the influence of game elements on motivation develops over time and additionally explore whether enjoyment influences motor performance. We describe two different studies that varied game elements in different exercises. Experiment 1 compared the durability of enjoyment for a gamified and a conventional balance exercise in elderly. Experiment 2 addressed the question whether adding game elements to a gait adaptability exercise enhances the durability of enjoyment and additionally tested whether the game elements influenced movement vigor and accuracy (motor performance). The results show that the game elements enhanced enjoyment. Enjoyment faded over time, but this decrease tended to be less pronounced in gamified exercises. There was no evidence that the game elements affected movement vigor or accuracy.

Keywords: motivation, pleasure, reward, psychomotor performance, video games, postural balance, gait, exercise

\section{INTRODUCTION}

Fred and Frank climb the Mount Everest. Fred is motivated for the hike because he will receive sponsor money when he reaches the top. Frank is motivated because he enjoys the activity of hiking. In other words: Fred is motivated by an extrinsic reward that is separable from the activity of hiking whereas Frank is motivated by an intrinsic reward that is inherent to the activity of hiking (Deci and Cascio, 1972). Task enjoyment is associated with intrinsic motivation (Ryan and Deci, 2017) which is considered a durable form of motivation that doesn't decrease much over time (Vansteenkiste et al., 2006). Consequently, there is a lot of enthusiasm about enhancing enjoyment in the context of rehabilitation. Moreover, enjoyment is an intrinsic form of reward and research in the field of motor learning has shown that rewards can enhance motor performance (Cheng et al., 2013; Reppert et al., 2015; Shadmehr et al., 2016; Summerside et al., 2018). Would Frank's enjoyment not only be durable but also influence how he performs the hike? In this paper, we investigate 
whether enjoyment of a rehabilitation exercise can be enhanced in a durable manner by adding game elements to the exercise, a process called "gamification" (Deterding et al., 2011) also known as "serious game" design or "persuasive game" design (Visch et al., 2013).

One reason why gamification has the power to enhance enjoyment is that it can the fulfill the basic psychological needs of autonomy, competence and relatedness described by Self Determination Theory (Ryan and Deci, 2000). Game worlds can foster Autonomy by setting aside "real-world" limitations. Moreover, digital games can offer a greater level of variety and player options and choice (Ryan and Deci, 2017). Offering free choice enhances experienced autonomy (Wulf et al., 2014). Similarly, the need for Self-Competence can be fulfilled by balancing of game difficulty and player skill ("flow") and by providing direct feedback on performance (Ryan et al., 2006; Przybylski et al., 2010). Gamification is thus suitable to enhance enjoyment. But before using it to increase the amount of exercise, it is important to understand the temporal development of the enjoyment.

The enjoyment may be durable because the psychological needs do not become satiated like physiological needs (Deci and Ryan, 2000). However, the extent to which an activity fulfills psychological needs may change over time. As a child, Frank may have felt competent walking to the park but as he has aged this activity probably no longer fulfilled his need for self-competence. Moreover, novelty has been proposed as an important cause of intrinsic motivation (White, 1959). If novelty is important, enjoyment may fade while novelty wears off. Indeed, the motivating influence of gamification has been found to decrease over time. In one study, school children's intrinsic motivation for performing video game exercises (exergames) during physical education faded over time (Sun, 2013). In another study use of a set of exergames decreased dramatically over time (Simons et al., 2015). What remains unknown is whether adding game elements, enhances the durability of enjoyment.

A second issue we raise here is whether Frank's enjoyment would influence how he performs the hike. There are two ways in which enjoyment may influence motor performance: by influencing movement vigor and by influencing movement accuracy. For extrinsic rewards such as money or food it is well established that they affect movement vigor (Shadmehr et al., 2016). Humans (Cheng et al., 2013; Reppert et al., 2015) make faster eye movements toward more rewarding stimuli. Similarly, reaching movements are faster toward more preferred candy (Sackaloo et al., 2015). In addition, one study showed that financial reward enhanced the accuracy of pointing movements under perturbed feedback (Gajda et al., 2016). One study even showed that reward can break the speed-accuracy trade-off (Fitts, 1954) making movements faster and more precise (Manohar et al., 2015). For intrinsic rewards such as those associated with enjoyment, their effect on motor performance is less clear. A few studies showed that positive feedback enhanced movement accuracy (Jourden et al., 1991; Bandura, 1997; Lewthwaite and Wulf, 2010). For instance, repeatedly telling participants that they performed above average - reduced sway in a balance task (Lewthwaite and Wulf, 2010). Also, framing a task as one that could be learnt instead of framing it as one that measures a basic ability improved performance (Jourden et al., 1991) and timeon-target in a pursuit rotor task (Bandura, 1997). Other studies report that performance was not enhanced by visual and audio design (Lohse et al., 2015), free choice of ball color (Wulf et al., 2014), or positive feedback resulting from loose success criteria (Ong and Hodges, 2017).

In the current paper, we describe two experiments that address the question whether game elements enhance the level and durability of enjoyment. Experiment 1 compared enjoyment between a commercially available gamified balance exercise a and conventional balance exercise. Experiment 2 compared enjoyment between a commercially available gait adaptability exercise and the same exercise with a number of game elements removed. In addition, Experiment 2 assessed the influence of game elements on movement vigor and accuracy. The experiments were not originally designed as parts of a single study. Because they do tell an overall story, they are presented together in this paper.

\section{EXPERIMENT 1: GAMIFIED VERSUS CONVENTIONAL BALANCE EXERCISE: INTRINSIC MOTIVATION}

Experiment 1 addressed the question whether gamification enhances the level and durability of enjoyment of balance exercise in elderly and tested three hypotheses. First, we hypothesized that the game elements would enhance enjoyment. Enjoyment probably decreases over time as motivation for exergames decreases over time (Sun, 2013; Simons et al., 2015). We therefore hypothesized that enjoyment decreases over time. As game elements induce enjoyment by fulfilling psychological needs that do not satiate (Ryan et al., 2006), we critically predict that game elements enhance the durability of enjoyment.

\section{Methods Design}

We used a between-subjects design in which we, in a pseudorandom order assigned half of the participants to the game group and the other half to the control group.

\section{Participants}

As the investigated balance training was designed for elderly users, participants $(N=28)$ were healthy community dwelling elderly (19 women and 9 men, age: $75.2 \pm 6.6$ years). Two participants dropped out during the study and were not included in the final analysis. Eventually, 12 participants took part in the game group and 12 participants took part in the control group. Participants were recruited from fitness classes offered for elderly and via the personal network of the experimenter. Inclusion criteria were: age 65 years or older, the ability to walk safely without assistance and/or assistive devices. Exclusion criteria were: neurological disorder, cardiopulmonary disorder and impaired vision after correction.

We used a single-blind parallel trial design in which participants from both groups were told that they would 
participate in a study comparing the efficacy of two types of balance training. Participants were recruited at fitness centers offering fitness classes for elderly. Participants who were recruited from the same fitness facility were assigned to the same experimental condition to keep participants blind to the experimental conditions. Duo's were allocated to one of the two experimental conditions in a random order with an allocation ratio of 1:1. Participants were tested at four locations in Netherlands: a fitness center in Utrecht, a community center in Utrecht and a community center in Graft and a second fitness center in Utrecht. Participants were tested at the location that was closest to where they were recruited.

The study was carried out in accordance with the recommendations of the Declaration of Helsinki and the ethical committee of the Faculty of Behavioural and Human Movement Sciences at the Vrije Universiteit Amsterdam. The protocol was approved by the ethical committee of the Faculty of Behavioural and Human Movement Sciences at the Vrije Universiteit Amsterdam. All subjects gave written informed consent in accordance with the Declaration of Helsinki.

\section{Materials Experiment 1}

Prior to starting the experimental test sessions participants were informed that they would participate in a study on balance training with the following text:

"Balance is essential to many daily activities. Balance declines with age and it can therefore be important to engage in balance training. The purpose of this study is to compare the effectivity of two different balance trainings."

The game group performed the "Garden hose game" which is a balance exercise designed by SilverFit BV that challenged the participant to maintain balance while placing the feet in varying positions. The game employed a screen and projector for visual display and a Kinect v2 for motion registration such that direct feedback on the participant's movements could be provided (Figure 1A). The screen displayed a garden hose in which two simultaneous target leaks could appear, together with a representation of the participant's feet. The task of the participant was to seal both leaks by placing a foot on each of them. When a leak had been sealed, the participant scored points, and two new leaks appeared. Game elements involved direct feedback on the stepping movements, scores for successful performance, audio and graphic design and a game narrative of sealing leaks in a garden hose. The exercise ended after $4 \mathrm{~min}$.

The control group performed a conventional balance course described in the literature as suitable for the target group (Pluchino et al., 2012). The balance course offered the following tasks in a fixed order (Figure 1B): (1) stepping on a compliant surface (the Sportbay ${ }^{\circledR}$ Balance Pad). (2) Walking over a narrow path indicated on the floor. (3) Turning around full cycle. (4) Backward walking. (5) Standing up from a chair with arms crossed over the chest. (6) Single leg stance. (7) Tandem walking. (8) Single leg swing. (9) Lifting a glass of water from the floor, walking while holding it, and placing it back on the ground. The balance course ended after $4 \mathrm{~min}$. If participants completed the course within 4 min a second round started.
Enjoyment was assessed using the Interest/Enjoyment scale of the Intrinsic Motivation Inventory (IMI, Ryan, 1982) and using a "Quick Motivation Index" (QMI) developed for the experiment. The English version of the IMI has adequate internal-consistency ( $\alpha=0.85$ ) (McAuley et al., 1989). We translated this version to Dutch to make it suitable for the elderly population. On the pen and paper questionnaire, participants indicated their agreement with several statements on a 7-point Likert scale. The QMI consisted two-items ratings in which participants were asked to respond vocally to the following two questions:

(1) On a scale of one to ten how much do you enjoy the task until now?

(2) On a scale of one to ten how motivated are you to continue?

\section{Procedure Experiment 1}

Participants were informed that balance exercises were repeated on three different days with a minimum interval of 1 week (Figure 1C) and that each session would take about $24 \mathrm{~min}$, consisting of three 4 -min exercise blocks interleaved by $4 \mathrm{~min}$ rest. Experimental sessions were performed in groups of two participants from the same experimental condition (game or control). The members of the duo alternately exercised and rested. Following each block participants responded to the two-item motivation ratings. At the end of the final session, participants completed the IMI.

\section{Data Analysis Experiment 1}

To assess the agreement between the QMI and the IMI as an indicator of the level of enjoyment, a Spearman rank-order correlation was calculated between the IMI Interest/Enjoyment subscale and QMI ratings. To further assess test-retest reliability of the QMI we calculated the intra-class correlation between the three test days for each block. Data analyses were aimed at testing two hypotheses, concerning the influence of gamification on enjoyment and concerning the temporal development of enjoyment.

(1) To test the hypothesis that a gamified exercise is more enjoyable than a conventional exercise, the QMI scores averaged over blocks were compared between the game and control group using a Mann-Whitney $U$-test.

(2) To test the hypothesis that enjoyment decreases over time we performed a Friedman rank order test on the QMI in the different blocks.

(3) To test the hypothesis that enjoyment was more durable in the game group than in the control group, we assessed whether the change in QMI from block 1 to 9

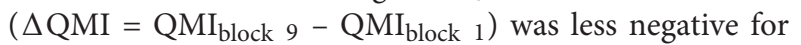
the game group compared to the control group using a one-sided Mann-Whitney $U$-test.

\section{Results Experiment 1}

The Interest/Enjoyment subscale was positively correlated with the rating of enjoyment $(r=0.75, p<0.001)$, rating of motivation to continue $(r=0.77, p<0.001)$ and QMI $(r=0.81, p<0.001$; Figure 2A). Moreover, a good reliability was found between the QMI measurements on different days. The average measure ICC 


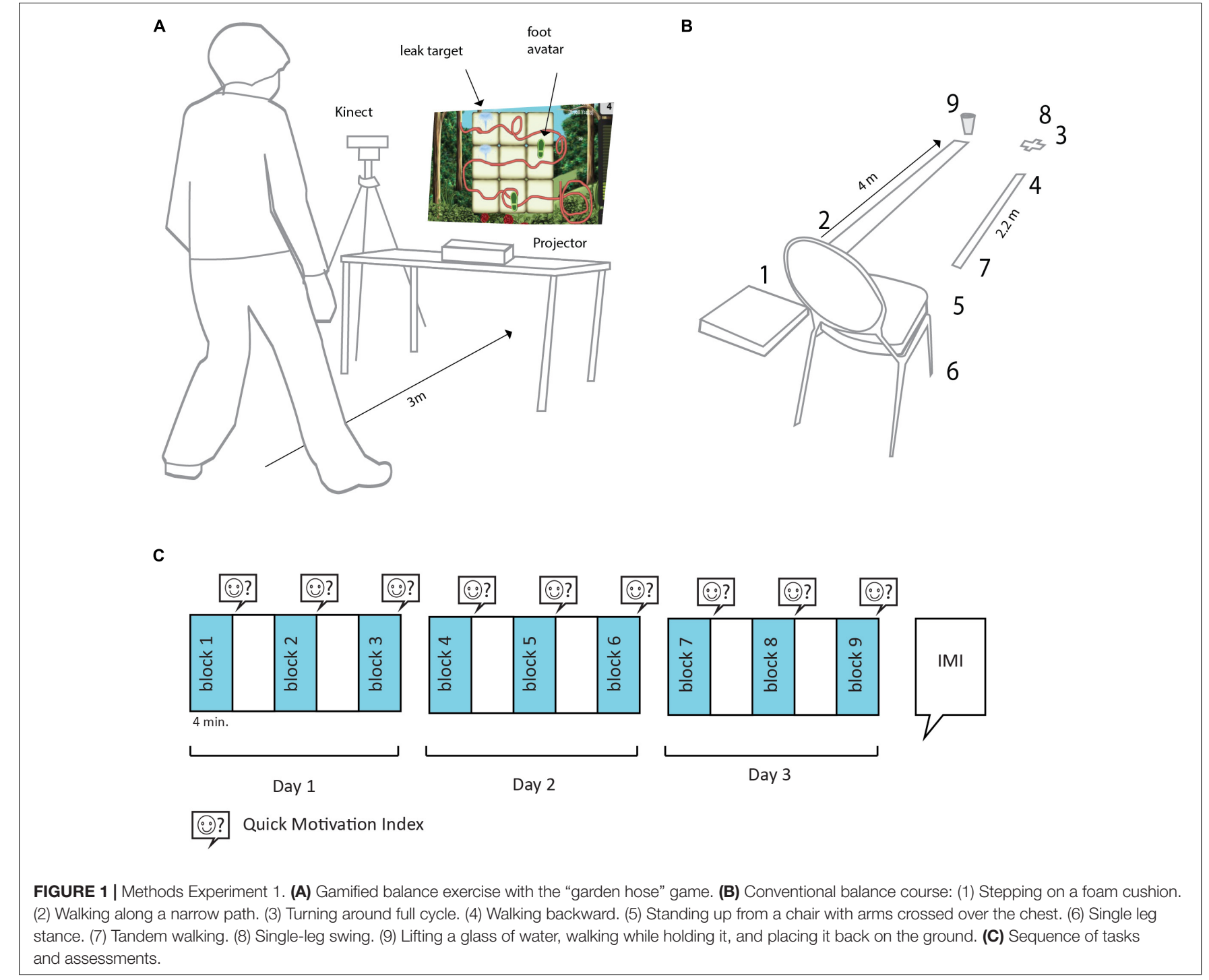

\section{B}

was 0.83 with a $95 \%$ confidence interval from 0.66 to 0.92 for block 1, 0.84 with a $95 \%$ confidence interval from 0.69 to 0.93 for block 2 and 0.92 with a $95 \%$ confidence interval from 0.84 to 0.96 for block 3 .

The game group scored higher on the IMI than the control group $[t(22)=-2.37, p=0.03$; Figure 2B]. The total QMI score over all blocks did not differ significantly between the game and control group $(U=40.5, z=1.82, p=0.068)$.

The durability of enjoyment was higher for the game group than for the control group $(U=38, z=-1.99, p=0.046$; Figure 2C). To analyze whether motivation changed in both groups, we performed two additional Friedman tests on the QMI in the control and game group $\left(\chi^{2}=29.04, p<0.001\right)$. In the control group, the QMI decreased over repeated blocks by $13 \%$ $\left(\chi^{2}=25.88, p<0.001\right)$. In the game group, in contrast, the QMI increased by $6 \%$ over repeated blocks $\left(\chi^{2}=22.85, p<0.001\right)$.

Post hoc power analyses showed that statistical power for the Mann-Whitney $U$-tests was low (0.64 for detecting a large effect size).
Thus, Experiment 1 showed that the QMI correlates with the Interest/Enjoyment subscale of the IMI. Most importantly, Experiment 1 showed that gamification affects the durability of enjoyment. In the control group, enjoyment decreased over time whereas in the game group enjoyment increased.

\section{EXPERIMENT 2: ADDED GAME ELEMENTS IN GAIT ADAPTABILITY EXERCISE: INTRINSIC MOTIVATION AND PERFORMANCE}

Experiment 2 addressed the question whether adding game elements to a gait adaptability exercise enhances the level and durability of exercise enjoyment. We hypothesized that game elements render a task more enjoyable and that enjoyment decreases over time. Because game elements create enjoyment by tapping into psychological needs that do not satiate (Ryan et al., 

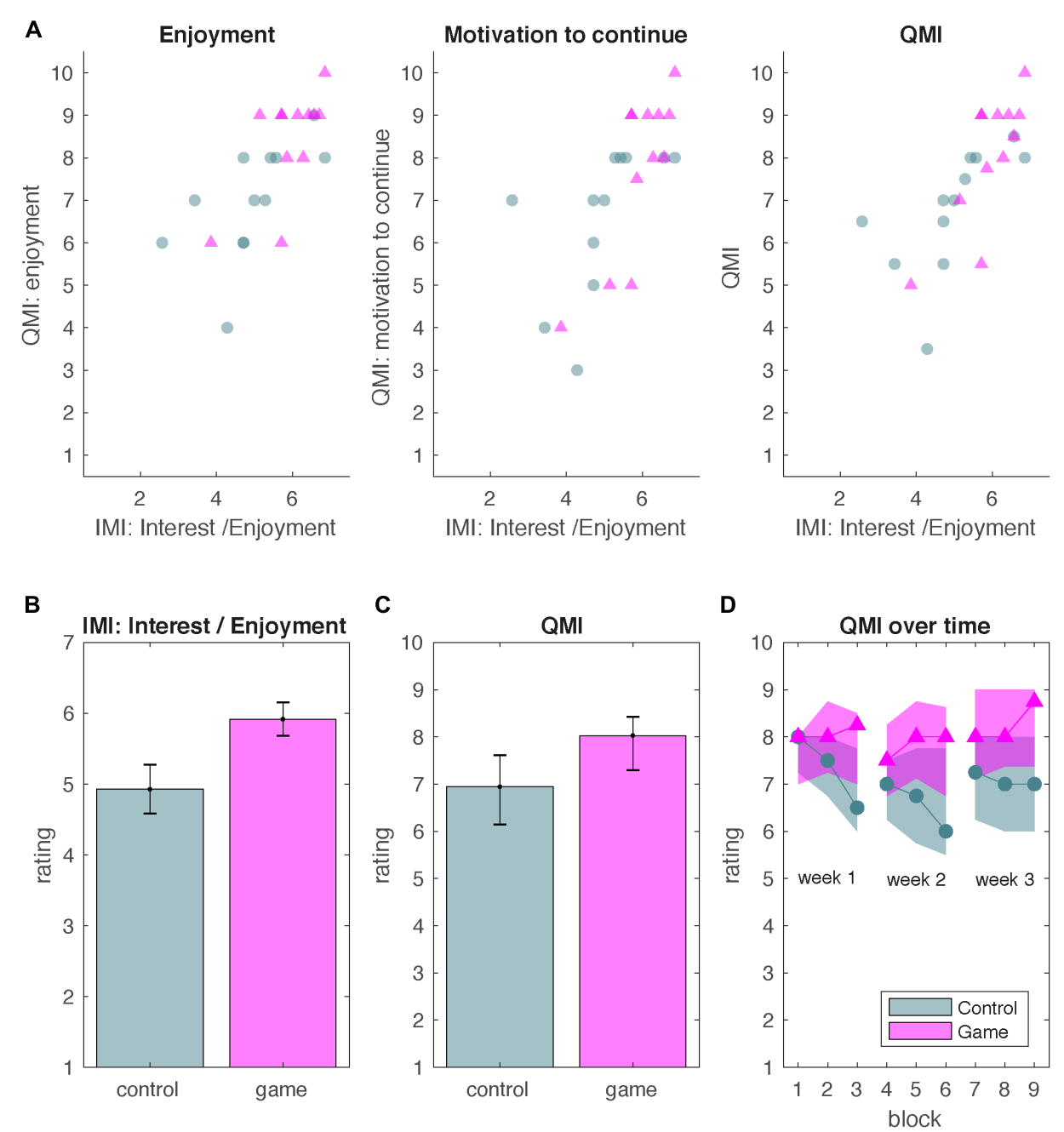

FIGURE 2 | Results Experiment 1. Intrinsic motivation for balance training. Green circles represent the control group and pink triangles represent the game group. (A) IMI Interest/Enjoyment subscale rating as a predictor of responses on the enjoyment rating, motivation-to-continue rating and Quick Motivation Index (QMI). (B) Mean IMI scores for the game and control group. Error bars represent standard errors of the mean. (C) Median QMI scores for the game and control group. Error bars represent the interquartile range. (D) Median QMI as a function of block for the game and control group. Shaded areas represent the interquartile range.

2006), we tested the hypothesis that game elements enhance the durability of enjoyment. In addition, based on the finding that rewards enhance movement vigor and perhaps also movement accuracy (Cheng et al., 2013; Reppert et al., 2015; Sackaloo et al., 2015; Shadmehr et al., 2016; Summerside et al., 2018) we explored whether the game elements enhanced movement vigor and accuracy.

\section{Methods Experiment 2 Design}

We used a parallel, single-blind trial in which, in a random order, half of participants was assigned to the game group and the other half to the control group. Participants were blinded to the experimental hypothesis by informing them that they would participate in a study investigating the effect of virtual feedback on gait rehabilitation.

\section{Participants}

We recruited forty-two healthy adults, mainly students Human Movement Sciences at the Vrije Universiteit (23 females and 19 males, age: $24.5 \pm 8.37$ years). Exclusion criteria were people who had experienced injuries that could affect their walking stability, who could not walk continuously for $30 \mathrm{~min}$, and who had received treadmill-walking therapy in the past or were familiar with Motekforce Link's gait adaptability game (Microbes). This study was carried out in accordance with the recommendations of the Declaration of Helsinki and the ethical committee of the Faculty of Behavioural and Human Movement Sciences at the Vrije Universiteit Amsterdam. The protocol was approved by the ethical committee of the Faculty of Behavioural and Human Movement Sciences at the Vrije Universiteit Amsterdam. All subjects gave written informed consent in accordance with the Declaration of Helsinki. Participants were tested at the Motekforce Link office in Amsterdam. 


\section{Materials}

The gait adaptability exercise was developed by Motekforce Link for the Gait Real-time Analysis Interactive Lab (GRAIL; Figure 3A). In the exercise participants walked on a treadmill while a virtual environment projected in front of them challenged them to catch virtual targets and avoid virtual obstacles that moved into the playing field from a random location outside the field. The targets could be caught and avoided by controlling the position of an avatar with the participant's center of mass (CoM) as tracked by the GRAIL system (Motekforce Link; for a video impression $\left.\mathrm{see}^{1}\right)$. The Microbes game is rich in game elements, taking advantage of the virtual environment to immerse the participant in a visually engaging fantasy world, balancing challenges to player skill, providing direct feedback using both audio and graphic effects and providing game scores. To ensure that the game challenged the healthy participants, we set the treadmill speed $10 \%$ above the preferred walking speed (Hak et al., 2012). The Microbes game consists of several minigames of which we selected three mini games (Figure 3B). The "Avoid Obstacles" (AO) mini game (Figure 3B; sec 4.20 in the video impression) was selected for a motivation pre- and posttest. The "Acceleration/Deceleration" (AD) mini game (sec 2.50 in the video impression), and Hit targets (HT) mini game (sec 1 in the video impression) were selected for the main experiment. For both the AD and HT mini game a control game was created from which all game elements that were expected to affect motivation but who had no direct influence on motor performance were removed. The following game elements were removed: visual display of scores, audio design, graphic design, visual display of the remaining time, number of caught targets, and the game narrative of an evolving Microbe.

Enjoyment was measured using the QMI that we also used in Experiment 1. The only difference being that we used a 7-point scale instead of a 10-point scale. Experiment 1 used a wider scale because this experiment was performed after Experiment 2 and aimed to achieve a higher QMI precision.

\section{Procedure}

The procedure is schematically depicted in Figure 3C. First, the participant's preferred walking speed was determined. For each participant the maximal comfortable treadmill walking speed (MCTWS) was determined based on a 1-up, 1-down staircase procedure with a step-size of $0.1 \mathrm{~m}$ per second described in detail by Dal et al. (2010). Next, participants were informed that they would perform a walking task that would last in total about $30 \mathrm{~min}$. They were also reminded that during the task they should remember that green objects (the targets) were good whereas red objects (the obstacles) were evil. No other instructions were provided. After that they performed the 5-min AO minigame pre-test. When the pre-test was finished, we explained the game narrative of an evolving Microbe to the participants in the game group: "You will be a microbe in a world of other bacteria and organisms. You live in a fictional world in which you need to collect life essence. Each time you have collected a life essence

\footnotetext{
${ }^{1}$ https://www.youtube.com/watch?v=bgV2-Tb6OJQ
}

you are closer to accomplishing your ultimate goal: developing and reaching the next level!"

After that, participants performed three 5-min blocks in which the AD and HT mini game alternated every time seven targets were caught within a game. After each block, the treadmill was stopped and the QMI was administered. After three blocks, another "AO" mini game was performed as a motivational post-test.

\section{Data Collection and Analysis}

Kinematic data on movement vigor and accuracy were based on the $2 \mathrm{D}$ position of the CoM avatar that the participant controlled with his or her center of mass. To this end, the CoM was tracked with the Vicon (Oxford, United Kingdom) motion capture system embedded in the GRAIL system and the $\mathrm{x}, \mathrm{y}$ position on the treadmill was calculated by the direct average of four reflective markers placed on the pelvis. Kinematic data were filtered offline in MATLAB 2017b using a two-way second-order Butterworth filter with a cut-off frequency of $2 \mathrm{~Hz}$ and were also analyzed using MATLAB.

Movement vigor was analyzed by the CoM avatar's velocity range (Vrange, Figure 3E), which was the 95\% range of the velocity of the CoM avatar within a block. This value reflects the range in which participants accelerated or decelerated in order to catch the targets and avoid the obstacles while the treadmill imposed a velocity that was constant.

Movement accuracy was measured from the CoM deviation (Figure 3D), which was the mean distance between the position of the CoM avatar and target position within a block. In the $\mathrm{AD}$ mini game, movement accuracy was calculated as the absolute one-dimensional distance between the $\mathrm{y}$ position of the CoM avatar and the middle of the target area. In the HT minigame, the movement accuracy was the absolute two-dimensional distance $(x, y$ position) between the CoM avatar and the center of the target circle.

Statistical tests were performed with SPSS version 24 and focused on 4 hypotheses testing the influence of game elements on motivation and testing the influence of motivation on movement vigor and movement accuracy:

\section{Motivation}

(1) To test the hypothesis that adding game elements increases task enjoyment, the overall QMI score was compared between the game and control group using a MannWhitney $U$-test.

(2) To test the hypothesis that adding game elements enhances the durability of enjoyment we tested whether the change in QMI between block 1 and 3 ( $\Delta \mathrm{QMI}$ ) was more negative for the control group compared to the game group using a one-sided Mann-Whitney $U$-test.

\section{Movement vigor}

(3) To test whether game elements influenced the movement vigor, we entered the CoM velocity range in the three blocks in a mixed ANOVA with group as a between-participants factor and block as a repeated factor. 
A

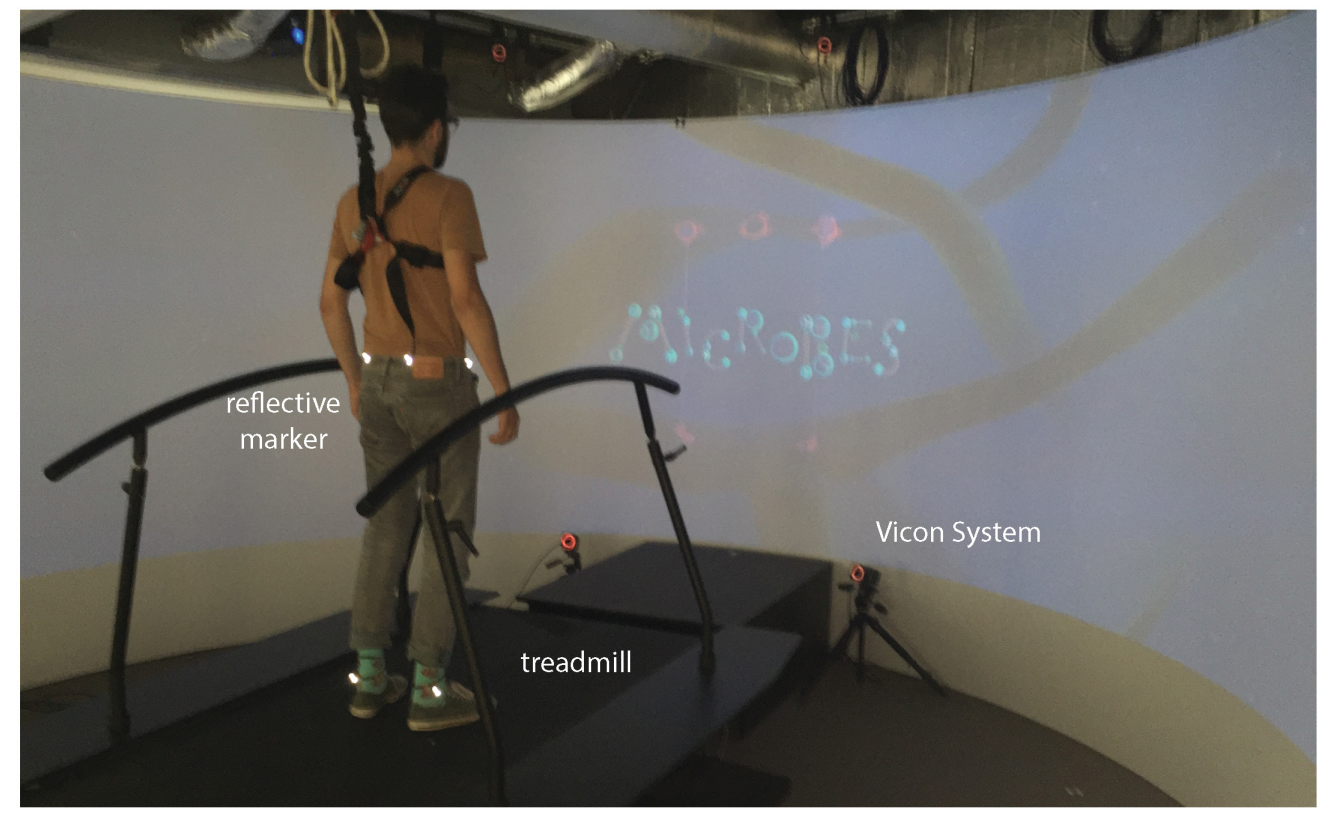

B
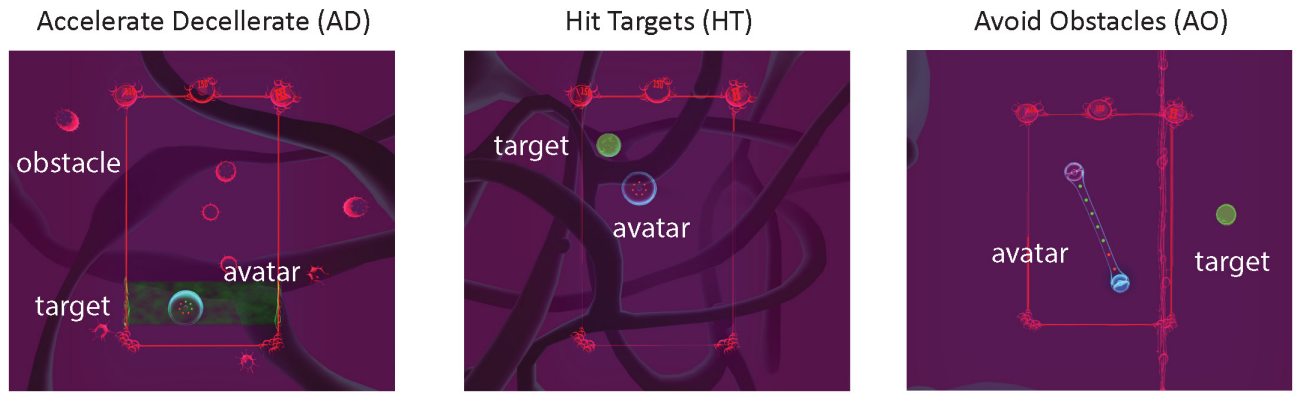

C

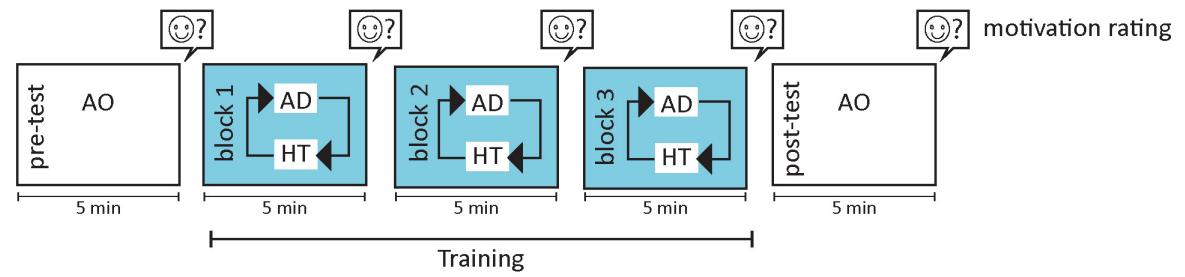

D

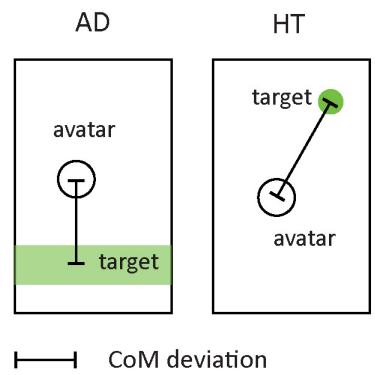

E

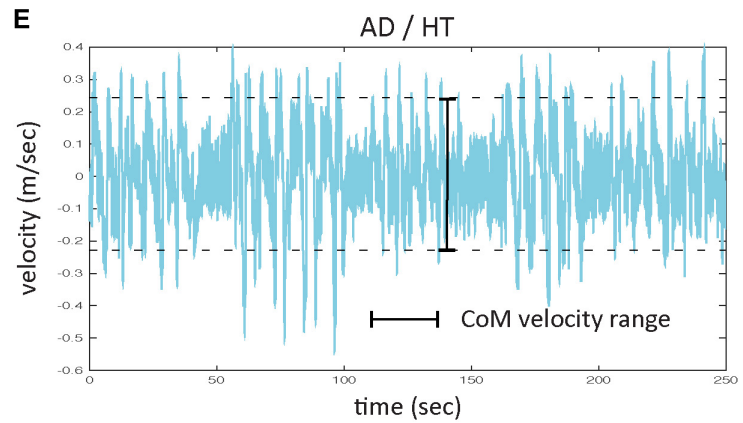

FIGURE 3 | Methods Experiment 2. (A) Gait Real-time Interactive Lab (GRAIL) system with treadmill, screen and Vicon system. (B) Microbes mini games with avatar, target and obstacles. (C) Sequence of tasks and assessments. (D) Movement accuracy was measured by the center of mass (CoM) deviation: the distance between the CoM avatar and target. (E) Movement vigor was measured from the $95 \%$ velocity range of the CoM avatar during a block. 
Movement accuracy

(4) To test whether game elements influenced movement accuracy we entered the CoM deviation data in the three blocks in a mixed ANOVA with group as a between participants factor and block as a repeated factor.

\section{Results Experiment 2 Enjoyment}

At pre-test, the QMI did not differ between groups, $U=373$, $z=-1.58, p=0.11$. Neither did the QMI differ between groups at post-test, $U=391, z=-1.06, p=0.29$. This indicates that differences between groups were caused by the difference in game elements during the three experimental blocks.

The Mann-Whitney $U$-test comparing the QMI averaged over the three experimental blocks between the game and control group showed that enjoyment was higher in the game group $($ Median $=6)$ compared to the control group $($ Median $=5)$, $U=104, z=-2.59, p=0.01$ (Figure 4A). The Friedman test comparing the QMI in block 1, 2, and 3 showed that the QMI decreased over blocks by $18 \%, \chi^{2}=38.26, p<0.001$. However, the decrease in enjoyment $(\triangle \mathrm{QMI})$ did not significantly differ between the game and control group, $U=138.5, z=-1.67$, $p=0.095$. Figure 4B does show a pattern consistent with Experiment 1: the decrease was less pronounced in the game group. Post hoc power analyses showed that statistical power for the Mann-Whitney $U$-tests was adequate only to detect a large effect size (power was 0.8 for detecting a large effect size).

\section{Movement Vigor}

The mixed ANOVA on the CoM velocity range in the game and control group (Figure 4C) showed no main effect of group, $F(1,37)=0.48, p=0.49$, no interaction of group and block, $F(2,74)=1.16, p=0.32$ and no main effect of block, $F(2,74)=1.99$, $p=0.14$. Thus, movement vigor did not differ between the game and control group.

\section{Movement Accuracy}

The mixed ANOVA on the CoM distance (Figure 4D) with group as a between-participants factor and with block as a repeated factor showed a main effect of block, $F(1.34,49.57)=15.46, p<0.001$, no main effect of group $F(1,37)=2.32, p=0.14$ and no interaction of group and block $F(1.34,49.57)=0.21, p=0.72$. Thus, there was no evidence that movement accuracy was higher in the game group.

Thus, Experiment 2 showed that gamification enhanced enjoyment. Although the data on the durability of enjoyment showed a pattern similar to Experiment 1, there was no significant effect of gamification on the durability of enjoyment. Game elements did not influence movement vigor or accuracy.

\section{GENERAL DISCUSSION}

This study investigated how the enjoyable effect of game elements on movement exercises develops over time. Are game elements an inexhaustible source of intrinsic reward or does enjoyment fade over time? Additionally, we also explored whether game elements enhance movement vigor and accuracy, as has been found for extrinsic reward (Haggard et al., 2000; Takikawa et al., 2002; Opris et al., 2011; Cheng et al., 2013; Manohar et al., 2015; Reppert et al., 2015; Sackaloo et al., 2015; Gajda et al., 2016). Overall, we found that the game elements increased enjoyment. Enjoyment, as measured with the QMI, decreased while participants repeatedly performed the same exercise (Experiment 1 and 2). However, in Experiment 1, enjoyment increased for participants who performed the gamified exercise. In Experiment 2 the decrease in motivation appeared to be attenuated for the game group, but there was no significant difference between the game and control group. There was no evidence that the game elements enhanced movement vigor or accuracy. In sum, our main finding is that gamification enhances the level and probably the durability of enjoyment.

\section{Limitations}

Before discussing the results any further, we need to emphasize two important limitations of the studies reported in this paper. First, we used a non-validated scale (QMI) that we developed for the experiments to measure the temporal decay of enjoyment. In addition, Experiment 1 used a non-validated Dutch Translation of the IMI. Therefore it remains to be established how the QMI relates to the construct of intrinsic motivation. A promising finding was that correlations between the QMI and the translated Interest/Enjoyment subscale of the IMI were adequate as were test-retest correlations for the QMI. Second, no a priori power analysis was performed, and sample sizes were only adequate to detect large effect sizes. For the influence of gamification on the level of enjoyment, the data were consistent across experiments, which adds evidence to the conclusion that the gamification increased the level and probably also the durability of intrinsic motivation. However, the finding that there was no relation between enjoyment and motor performance is difficult to interpret. Thus, the results should be read as preliminary evidence that can be used to design future studies.

\section{The Influence of Game Elements on Enjoyment}

Our study confirms the idea that gamification can increase enjoyment of a task (for reviews see Connolly et al., 2012; Hamari et al., 2014). Experiment 1 showed that enjoyment of a balance exercise was higher for participants who performed a gamified exercise that involved sealing leaks with one's footsteps than for participants who performed a conventional balance course consisting of a set of nine different tasks (see methods Experiment 1). As the movement tasks differed between the gamified and conventional training, the difference in enjoyment may have been related to the task rather than to the game elements in the gamified exercise (scores, aesthetic design, direct feedback, narrative). Experiment 2 tested whether the removal of game elements from a gamified gait adaptability exercise reduced enjoyment of this exercise. We found that the participants who performed the exercise without additional game elements (scores, 

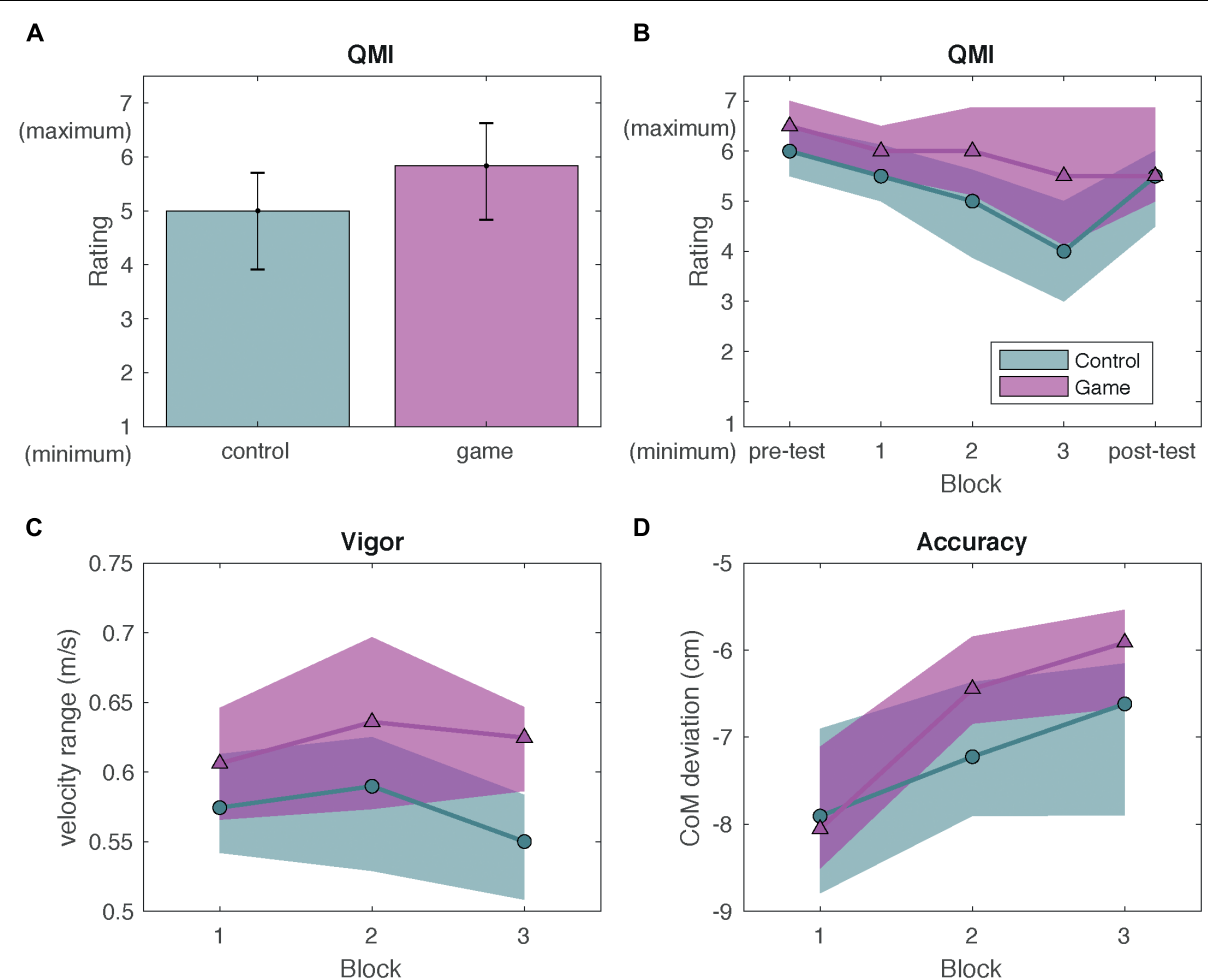

D

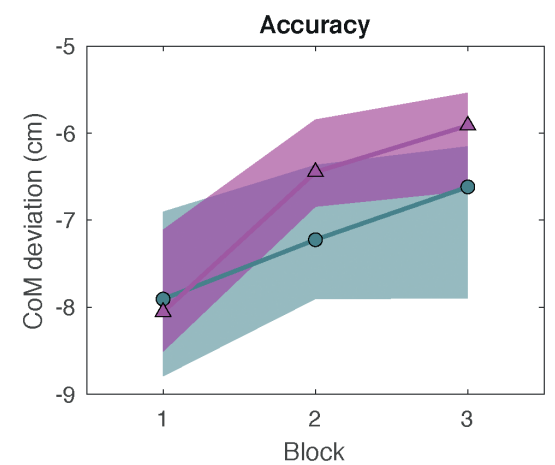

FIGURE 4 | Results Experiment 2. Green circles represent data from the control group and pink triangles represent data from the game group. Shaded areas indicate the interquartile range. (A) Median QMI for the game and control group. Error bars represent the interquartile range. (B) Median QMI with interquartile range as a function of block. (C) Median movement vigor with interquartile range as a function of block. (D) Median movement accuracy with interquartile range as a function of block.

aesthetic design, narrative) indeed experienced the exercise as less enjoyable.

Enjoyment can be considered an intrinsic form of reward that evokes motivation. Consistent with this idea, we measured enjoyment by asking both about enjoyment directly and by asking about motivation to continue and found that responses on the two items were highly correlated (Figure 2A). This suggests that when motivation needs to be enhanced, creating intrinsically rewarding games provides an alternative to using extrinsic rewards and punishments such as financial incentives.

A potential benefit of using gamification to enhance motivation is that it might stimulate an intrinsic type of motivation in which the motivation is perceived as internally regulated. The benefit of evoking intrinsic motivation is that intrinsic motivation has been associated with greater persistence at a task than extrinsic motivation in which the motivation is perceived as externally regulated (for a review see Deci et al., 1999). Moreover, intrinsic motivation has been proposed to result in higher quality performance than extrinsic motivation (Wulf and Lewthwaite, 2016; Ryan and Deci, 2017). Whereas intrinsic rewards foster intrinsic motivation, extrinsic rewards tend to reduce the intrinsic type of motivation (Deci et al., 1999; Ryan and Deci, 2017) although there has been some controversy on this subject (Cameron and Pierce, 1994). Thus, gamification may benefit the durability of motivation by increasing intrinsic motivation. Off course, gamification would only result in durable motivation if the enjoyment of the gamification is durable. In the next paragraphs we consider the temporal decay of enjoyment and whether there was a relation with motor performance.

\section{The Durability of Intrinsic Motivation}

Using a novel QMI, we showed the expected decrease in enjoyment over time. Interestingly, for the gamified exercises enjoyment increased over time (Experiment 1) or the decrease tended to be attenuated (Experiment 2). This is a clear indication that game elements enhance the durability of enjoyment, as opposed to studies that show that motivation for exergames decreases over time (Sun, 2013; Simons et al., 2015). Game elements may enhance the durability of enjoyment because the enjoyment results from the fulfillment of needs that do not satiate (Ryan et al., 2006). In contrast to conventional exercise, game elements can create myriad opportunities for fostering these needs, for instance providing positive feedback to enhance competence and offering choice to foster autonomy. The attenuation of the decrease was statistically significant following the nine 5-min blocks of Experiment 1 but not following the three 5-min blocks of Experiment 2. Reported effects of rewards on motivation have been small (Rummel, 1988). It is thus wellpossible that the absence of a statistically significant effect in Experiment 2 was due to a lack of statistical power. The longest timescale on which we assessed intrinsic motivation was 3 weeks. This is a much shorter timescale than the timescale on which 
intrinsic motivation is needed in for instance a rehabilitation or sports setting. How the influence of game elements on intrinsic motivation develops over longer timescales is an important question for future research.

\section{Intrinsic Motivation and Movement Vigor and Accuracy}

Although we observed that game elements influenced the level of enjoyment, creating intrinsic reward, we did not find that they enhanced movement vigor or accuracy as has been shown for extrinsic reward (Haggard et al., 2000; Takikawa et al., 2002; Opris et al., 2011; Cheng et al., 2013; Manohar et al., 2015; Reppert et al., 2015; Sackaloo et al., 2015; Gajda et al., 2016). It is possible that we did not observe an effect of the gamification on movement vigor or accuracy because our study lacked the statistical power to detect such an effect. Effects of rewards on motor performance are often small, especially for intrinsic rewards such as scored points (Summerside et al., 2018) or faces (Xu-Wilson et al., 2009) and influences on learning are highly variable (Galea et al., 2015; van der Kooij and Overvliet, 2016; van der Kooij et al., 2018). It is to be expected that effects of enjoyment are similarly small. Moreover, measuring enjoyment with self-reports may lack sensitivity. Besides by using a larger sample size, future studies on the influence of enjoyment on motor performance could improve statistical power by increasing measurement sensitivity and by capitalizing on the passage of time as a factor that increases between-group differences in enjoyment.

\section{Suggestions for Future Research}

To gain a better understanding of how and when gamification benefits real-world behavior, time sensitive measurements of outcome variables such as motivation need to be developed (van der Kooij et al., 2015). Using the novel QMI our study suggests that a benefit of gamification is that it enhances the durability of enjoyment and thereby intrinsic motivation. To our knowledge, no validated and suitable tool was available to assess enjoyment repeatedly without interfering with the gamified exercise. Although the results look promising, a number of methodological improvements may be made in the QMI. First, sensitivity and options for statistical analyses could be improved by collecting continuous rather than ordinal data. For instance by using a magnitude estimation protocol (Stevens, 1975). Second, we used verbal reports to avoid interference with the movement tasks. Validity of the QMI may be improved by using anonymous ratings that are incorporated in the game software instead of oral responses which may be biased by socially desirable answers. An interesting question for future research is whether including both a question on enjoyment and a question on motivation to continue provides an assessment of enjoyment-based intrinsic motivation or whether these two questions address different aspects of motivation.

Another interesting topic for future research is whether influences of gamification on enjoyment and motor performance differ between patients and healthy participants, such as tested in the current study. For instance, influences of gamification on enjoyment may be different because healthy participants and patients are motivated by different types of rewards. The healthy participants participated voluntarily. They may have intended to help the experimenter, contribute to science or may have been curious. Consistent with the relatively high baseline level of enjoyment for both the game and control group (Figures 2D, 4B), the healthy participants may have been largely motivated by rewards that are intrinsic to the activity of participating although participants in Experiment 2 may also have participated for the financial compensation. Patients, in contrast, are generally required to perform their rehabilitation exercises and may have be largely motivated by reward extrinsic to the exercise, for instance faster recovery. Influences of gamification on enjoyment may be larger for a group with a lower baseline enjoyment. Gamification may also affect motor performance differently for patients and healthy participants. Baseline levels of motor performance will be lower, perhaps allowing for a greater influence of gamification on motor performance (Deci et al., 1999; Eisenberger et al., 1999; Summerside et al., 2018).

\section{CONCLUSION}

Adding game elements to a task enhances the level of enjoyment and causes the enjoyment to fade slower over time than it would otherwise. There was no evidence that enjoyment led to better motor performance. An interesting avenue for future research would be to develop paradigms that use the influence of time to study motor performance under varying levels of enjoyment. This way we can develop a deeper insight into whether Frank's enjoyment would affect how he performs the hike.

\section{AUTHOR CONTRIBUTIONS}

KvdK, RvD, MvV, CdW, KO, and FS wrote the manuscript. $\mathrm{KvdK}, \mathrm{RvD}$, and $\mathrm{MvV}$ analyzed the data and collected the data. KvdK designed the Experiment 1. CdW, RvD, MvV, KO, and KK designed the Experiment 2.

\section{FUNDING}

The research was funded by Stichting Technologie en Wetenschap (STW) Open Technologie Programma (OTP) grant 12668 awarded to Jeroen Smeets. KO was additionally supported by a Marie-Curie IEF grant by the European Commission (624297).

\section{ACKNOWLEDGMENTS}

We thank SilverFit for providing the SilverFit equipment and software used in Experiment and for providing technical support. We also thank Jasper van de Lagemaat, Simone van Wezel, Pieter van Doorn, and Theo Zondag for testing participants. 


\section{REFERENCES}

Bandura, A. (1997). Self-Efficacy: The Exercise of Control. New York, NY: Freeman. Cameron, J., and Pierce, W. D. (1994). Reinforcement, reward, and intrinsic motivation: a meta-analysis. Rev. Educ. Res. 64, 363-423. doi: 10.3102/ 00346543064003363

Cheng, L. L., Hung, L. Y., Quinet, J., and Kosek, K. (2013). Cognitive regulation of saccadic velocity by reward prospect. Eur. J. Neurosci. 38, 2434-2444. doi: 10.1111/ejn.12247

Connolly, T. M., Boyle, E. A., MacArthur, E., Hainey, T., and Boyle, J. M. (2012). A systematic literature review of empirical evidence on computer games and serious games. Comput. Educ. 59, 661-686. doi: 10.1016/j.compedu.2012.03.004

Dal, U., Erdogan, T., Resitoglu, B., and Beydagi, H. (2010). Determination of preferred walking speed on treadmill may lead to high oxygen cost on treadmill walking. Gait Posture 31, 366-369. doi: 10.1016/j.gaitpost.2010.01.006

Deci, E. L., and Cascio, W. F. (1972). Changes in intrinsic motivation as a function of negative feedback and threats. Paper Presented at the Meeting of the Eastern Psychological Association, Boston, MA.

Deci, E. L., Koestner, R., and Ryan, R. M. (1999). A meta-analytic review of experiments examining the effects of extrinsic rewards on intrinsic motivation. Psychol. Bull. 125, 627-668; discussion 692-700. doi: 10.1037/0033-2909. 125.6.627

Deci, E. L., and Ryan, R. M. (2000). The what and why of goal pursuits: human needs and the self-determination of behavior. Psychol. Inq. 11, 227-268. doi: 10.1207/S15327965PLI1104_01

Deterding, S., Dixon, D., Khaled, R., and Nacke, L. (2011). "From game design elements to gamefulness: defining "gamification", in Proceedings of the 15th International Academic MindTrek Conference: Envisioning Future Media Environments (New York, NY: ACM), 9-15. doi: 10.1145/2181037.218 1040

Eisenberger, R., Pierce, W. D., and Cameron, J. (1999). Effects of reward on intrinsic motivation - negative, neutral, and positive: comment on Deci, Koestner, and Ryan (1999). Psychol. Bull. 125, 677-691. doi: 10.1037/0033-2909.125. 6.677

Fitts, P. M. (1954). The information capacity of the human motor system in controlling the amplitude of movement. J. Exp. Psychol. 47, 381-391. doi: 10. 1037/h0055392

Gajda, K., Sulzenbruck, S., and Heuer, H. (2016). Financial incentives enhance adaptation to a sensorimotor transformation. Exp. Brain Res. 10, 2859-2868. doi: 10.1007/s00221-016-4688-3

Galea, J. M., Mallia, E., Rothwell, J., and Diedrichsen, J. (2015). The dissociable effects of punishment and reward on motor learning. Nat. Neurosci. 18, 597602. doi: $10.1038 / \mathrm{nn} .3956$

Haggard, P., Newman, C., Blundell, J., and Andrew, H. (2000). The perceived position of the hand in space. Percept. Psychophys. 62, 363-377. doi: 10.3758/ BF03205556

Hak, L., Houdijk, H., Steenbrink, F., Mert, A., and van der Wurff, P. (2012). Speeding up or slowing down?: gait adaptations to preserve gait stability in response to balance perturbations. Gait Posture 36, 260-264. doi: 10.1016/j. gaitpost.2012.03.005

Hamari, J., Koivisto, J., and Sarsa, H. (2014). "Does gamification work? - A literature review of empirical studies on gamification," in Proceedings of the 47th Hawaii International Conference on System Sciences (Waikoloa, HI: IEEE), 3025-3034. doi: 10.1109/HICSS.2014.377

Jourden, F. J., Bandura, A., and Banfield, J. T. (1991). The impact of conceptions of ability on self-regulatory factors and motor skill acquisition. J. Sport Exerc. Psychol. 8, 213-226. doi: 10.1123/jsep.13.3.213

Lewthwaite, R., and Wulf, G. (2010). Social-comparative feedback affects motor skill learning. Q. J. Exp. Psychol. 63, 738-749. doi: 10.1080/1747021090311 1839

Lohse, K., Boyd, L., and Hodges, N. (2015). Engaging environments enhance motor skill learning in a computer gaming task. J. Mot. Behav. 48, 172-182. doi: 10.1080/00222895.2015.1068158

1Manohar, S. G., Chong, T. T. J., Apps, M. A. J., Batla, A., Stamelou, M., Jarman, P., et al. (2015). Reward pays the cost of noise reduction in motor and cognitive control. Curr. Biol. 25, 1707-1716. doi: 10.1016/j.cub.2015. 05.038
McAuley, E., Duncan, T., and Tammen, V. V. (1989). Psychometric properties of the intrinsic motivation inventory in a competitive sport setting: a confirmatory factor analysis. Res. Q. Exerc. Sport 60, 48-58. doi: 10.1080/02701367.1989. 10607413

Ong, N. T., and Hodges, N. J. (2017). Balancing our perceptions of the efficacy of success-based feedback manipulations on motor learning. J. Mot. Behav. 235, 3605-3618. doi: 10.1080/00222895.2017.1383227

Opris, I., Lebedev, M., and Nelson, R. J. (2011). Motor planning under unpredicatable reward: modulations of movement vigor and primate striatum activity. Front. Hum. Neurosci. 5:e65. doi: 10.3389/fnins.2011. 00061

Pluchino, A., Lee, S. Y., Asfour, S., Roos, B. A., and Signorile, J. F. (2012). Pilot study comparing changes in postural control after training using a video game balance board program and 2 standard activity-based balance intervention programs. Arch. Phys. Med. Rehabil. 93, 1138-1146. doi: 10.1016/j.apmr.2012. 01.023

Przybylski, A. K., Rigby, C. S., and Ryan, R. M. (2010). A motivational model of video game engagement. Rev. Gen. Psychol. 14, 154-166. doi: 10.1037/a001 9440

Reppert, T. R., Lempert, K. M., Glimcher, P. W., and Shadmehr, R. (2015). Modulation of saccade vigor during value-based decision making. J. Neurosci. 35, 15369-15378. doi: 10.1523/JNEUROSCI.2621-15.2015

Rummel, A. (1988). Cognitive evaluation theory: a meta-analytic review of the literature. Soc. Behav. Pers. 16, 147-164. doi: 10.1186/1471-243113-172

Ryan, R. M. (1982). Control and information in the intrapersonal sphere: an extenSion of cognitive evaluation theory. J. Pers. Soc. Psychol. 43, 450-461. doi: 10.1037/0022-3514.43.3.450

Ryan, R. M., and Deci, E. L. (2000). Intrinsic and extrinsic motivations: classic definitions and new directions. Contemp. Educ. Psychol. 25, 54-67. doi: 10.1006/ ceps. 1999.1020

Ryan, R. M., and Deci, E. L. (2017). Self-Determination Theory. Basic Psychological Needs in Motivation, Development and Wellness. New York, NY: The Guilford Press.

Ryan, R. M., Rigby, C. S., and Przybylski, A. (2006). The motivational pull of video games: a self-determination theory approach. Motiv. Emot. 30, 347-363. doi: 10.1007/s11031-006-9051-8

Sackaloo, K., Strouse, E., and Rice, M. S. (2015). Degree of preference and its influence on motor control when reaching for most preferred, neutrally preferred, and least preferred candy. OTJR 35, 81-88. doi: 10.1177/ 1539449214561763

Shadmehr, R., Huang, H. J., and Ahmed, A. A. (2016). A representation of effort in decision-making and motor control. Curr. Biol. 26, 1929-1934. doi: 10.1016/j. cub.2016.05.065

Simons, M., Brug, J., Chinapaw, M. J. M., de Boer, M., Seidell, J., and de Vet, E. (2015). Replacing non-active video gaming by active video gaming to prevent excessive weight gain in adolescents. PLoS One 10:e126023. doi: 10.1371/ journal.pone.0126023

Stevens, S. S. (1975). Psychophysics: Introduction to Its Perceptual, Neural and Social Prospects. New York, NY: John Wiley.

Summerside, E. M., Shadmehr, R., and Ahmed, A. (2018). Vigor of reaching movements: reward discounts the cost of effort. J. Neurophysiol. 119, 23472357. doi: 10.1152/jn.00872.2017

Sun, H. (2013). Impact of exergames on physical activity and motivation in elementary school students: a follow-up study. J. Sport Health Sci. 2, 138-145. doi: 10.1016/j.jshs.2013.02.003

Takikawa, Y., Kawagoe, R., Itoh, H., Nakahara, H., and Hikosaka, O. (2002). Modulation of saccadic eye movements by predicted reward outcome. Exp. Brain Res. 142, 284-291. doi: 10.1007/s00221-0010928-1

van der Kooij, K., Hoogendoorn, E., Spijkerman, R., and Visch, V. T. (2015). Validation of games for behavioral change: connecting the playful and serious. Int. J. Serious Games 2, 63-76. doi: 10.17083/ijsg.v2i3.75

van der Kooij, K., Oostwoud-Wijdenes, L., Rigterink, T., Overvliet, K. E., and Smeets, J. B. J. (2018). Reward abundance interferes with error-based learning in a visuomotor adaptation task. PLoS One 13:e0193002. doi: 10.1371/journal. pone.0193002 
van der Kooij, K., and Overvliet, K. E. (2016). Rewarding imperfect motor performance reduces adaptive changes. Exp. Brain Res. 234, 1441-1450. doi: 10.1007/s00221-015-4540-1

Vansteenkiste, M., Lens, W., and Deci, E. L. (2006). Intrinsic versus extrinsic goal contents in self-determination theory: another look at the quality of academic motivation. Educ. Psychol. 41, 19-31. doi: 10.1207/s15326985ep4101_4

Visch, V. T., Vegt, N., Anderiessen, H., and van der Kooij, K. (2013). "Persuasive game design: a model and its definitions," in Proceedings of the CHI 2013: Workshop Designing Gamification: Creating Gameful and Playful Experiences (Paris: ACM).

White, R. W. (1959). Motivation reconsidered: the concept of competence. Psychol. Rev. 66, 297-333. doi: 10.1037/h0040934

Wulf, G., Chiviacowsky, S., and Lopes Cardozo, P. (2014). Additive benefits of autonomy support and enhanced expectancies for motor learning. Hum. Mov. Sci. 37, 12-20. doi: 10.1016/j.humov.2014.06.004

Wulf, G., and Lewthwaite, R. (2016). Optimizing performance through intrinsic motivation and attention for learning: the OPTIMAL theory of motor learning. Psychon. Bull. Rev. 23, 1382-1414. doi: 10.3758/s13423-015-0999-9
Xu-Wilson, M., Zee, D., and Shadmehr, R. (2009). The intrinsic value of visual information affects saccade velocities. Exp. Brain Res. 196, 475-481.

Conflict of Interest Statement: CdW and FS are employed by Motekforce Link, the company that developed the gamified gait adaptability exercise that we used in Experiment 2.

The remaining authors declare that the research was conducted in the absence of any commercial or financial relationships that could be construed as a potential conflict of interest.

Copyright (c) 2019 van der Kooij, van Dijsseldonk, van Veen, Steenbrink, de Weerd and Overvliet. This is an open-access article distributed under the terms of the Creative Commons Attribution License (CC BY). The use, distribution or reproduction in other forums is permitted, provided the original author(s) and the copyright owner(s) are credited and that the original publication in this journal is cited, in accordance with accepted academic practice. No use, distribution or reproduction is permitted which does not comply with these terms. 